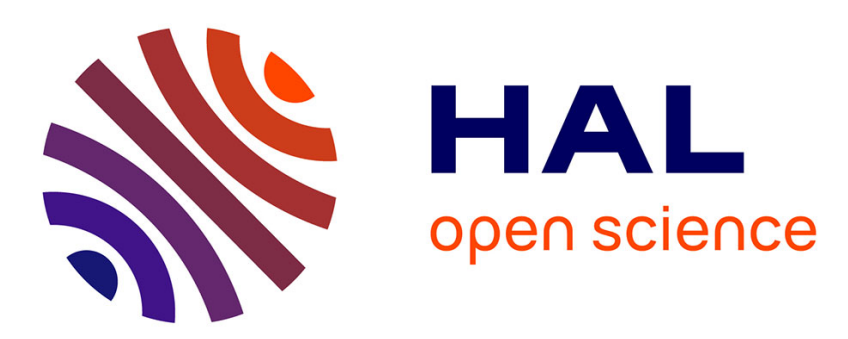

\title{
Determination of the Singlet-Triplet Separation of H2 by a "Level-Anticrossing" Technique
}

\author{
R. Jost, M. Lombardi
}

\section{To cite this version:}

R. Jost, M. Lombardi. Determination of the Singlet-Triplet Separation of H2 by a "Level-Anticrossing" Technique. Physical Review Letters, 1974, 33, pp.53-56. 10.1103/PHYSREVLETT.33.53 . hal00974331

\section{HAL Id: hal-00974331 \\ https://hal.science/hal-00974331}

Submitted on 6 Apr 2014

HAL is a multi-disciplinary open access archive for the deposit and dissemination of scientific research documents, whether they are published or not. The documents may come from teaching and research institutions in France or abroad, or from public or private research centers.
L'archive ouverte pluridisciplinaire HAL, est destinée au dépôt et à la diffusion de documents scientifiques de niveau recherche, publiés ou non, émanant des établissements d'enseignement et de recherche français ou étrangers, des laboratoires publics ou privés. 


\title{
PHYSICAL REVIEW LETTERS
}

\begin{tabular}{lcc}
\hline \hline Volume 33 & 8 JULY 1974 & Number 2 \\
\hline \hline
\end{tabular}

\section{Determination of the Singlet-Triplet Separation of $\mathrm{H}_{2}$ by a "Level-Anticrossing" Technique*}

\author{
R. Jost and M. Lombardi \\ Laboratoire de Spectrométrie Physique, $\dagger$ Université Scientifique et Médicale de Grenoble, \\ 38041 Grenoble Cedex, France \\ (Received 13 May 1974)
}

\begin{abstract}
We show that the singlet-triplet separation of $\mathrm{H}_{2}$ must be lowered by $157.7 \pm 0.2 \mathrm{~cm}^{-1}$ compared to the hitherto accepted value of Dieke. We have used a new kind of "level anticrossing" method in which the anticrossing is due to the coupling of a singlet and a triplet state by spin-orbit or hyperfine Fermi contact interaction.
\end{abstract}

Hydrogen, $\mathrm{H}_{2}$, is the simplest of the neutral molecules, and is therefore a good test for fundamental molecular calculations. Very refined calculations have been made by Kolos and Wolniewicz ${ }^{1}$ : They achieved an accuracy of a few inverse centimeters in the calculation of the location and properties of singlet energy levels. When applied to triplet levels, these calculations, confirming older and less precise ones of Rothenberg and Davidson, ${ }^{2}$ have raised a problem ${ }^{3}$ : The accepted experimental value of the separation of singlet and triplet levels is apparently some 150 $\mathrm{cm}^{-1}$ too great. However, the experimental situation is not clear: Separation within singlet and triplet levels is determined with the usual accuracy by optical spectroscopy but, since $\mathrm{H}_{2}$ has a very good Russell-Saunders spin coupling scheme, no transition between singlet and triplet levels has been identified and so the relative position of these two groups of levels is uncertain. The experimental separation of singlet and triplet levels in $\mathrm{H}_{2}$ is based on an uncertain determination of ionization potentials by extrapolation of the Rydberg series. There have been variations in this assignment: The now generally accepted results of Dieke, ${ }^{4}$ which we shall use as a basis in this Letter, are $141 \mathrm{~cm}^{-1}$ higher than the older ones of Beutler and Jünger. ${ }^{5}$ Recently, Herzberg and Jungen ${ }^{6}$ have obtained by absorption in the uv the ionization potential of singlet states of $\mathrm{H}_{2}$ with better precision.

To see if the anticrossing experiment was possible we had to find pairs of singlet-triplet levels whose separation did not exceed $10 \mathrm{~cm}^{-1}$ (because of magnetic-field limitation). Assuming, as is the feeling of most of the specialists we know, that the theoretical results are more reliable than Dieke's experimental ones we have looked for another experimental determination of this separation. The method is based upon comparison of $3 d$ and $4 d$ levels in $\mathrm{H}_{2}$ and in $\mathrm{He}$ (there is a singlet-triplet line in $\mathrm{He}$ ) since $\mathrm{He}$ is the unitedatom limit of $\mathrm{H}_{2}$, and since in both cases there is a large hydrogenoid outer $3 d$ and $4 d$ orbit around a small core.

The separation of $3 d^{1} D$ and $3 d^{3} D$ levels of He is $3.32 \mathrm{~cm}^{-1}$ and the separation of $4 d^{1} D$ and $4 d$ ${ }^{3} D$ is $1.88 \mathrm{~cm}^{-1}$. In $\mathrm{H}_{2}$ we have selected $3 d \Pi^{-}$, $4 d \Pi^{-}, 3 d \Delta^{-}$levels; $3 d$ and $4 d \Sigma, \Pi^{+}$, and $\Delta^{+}$ levels are perturbed differently and the singlettriplet separation of a pair of levels with the same $N$ and $v$ is somewhat erratic. Within $\Pi^{-}$or $\Delta^{*}$ levels, on the contrary, there is great regularity and a weak and smooth dependence on $v$. The singlet-triplet separation in Dieke's basis, extrapolated to rotational zero and $v=-\frac{1}{2}$, is re- 
spectively $-149,-153,-145$, and $-153 \mathrm{~cm}^{-1}$ (uncertain) for $3 d \Pi^{-}, 4 d \Pi^{-}, 3 d \Delta^{-}$, and $4 d \Delta^{\circ}$. This raises immediately the following question: Why are all these separations of the order of $-150 \mathrm{~cm}^{-1}$ instead of plus a few inverse centimeters as in He? If we suppose that the ratio of singlet-triplet separations between $n=3$ and $n=$ 4 is the same in $\mathrm{H}_{2}$ and $\mathrm{He}$, it follows that triplets must be lowered by $158 \mathrm{~cm}^{-1}$ for $\Pi^{-}$and 162 $\mathrm{cm}^{-1}$ (uncertain) for $\Delta^{-}$levels. We then assume provisionally a lowering of $158 \mathrm{~cm}^{-1}$ of the triplet levels. In this hypothesis, therefore, we choose for our study three pairs of levels, $3 d$ ${ }^{1-3} \Sigma, v=0, N=2$ and 3 , and the $3 d^{1-3} \Delta, v=1$, $N=3$ levels, which are less than $5 \mathrm{~cm}^{-1}$ apart.

The general form of "anticrossing" experiments has been described by Wieder and Eck. ${ }^{7}$ In the "pure anticrossing" case, two radiating levels are populated differently by various excitation mechanisms, and the mechanism of detec-

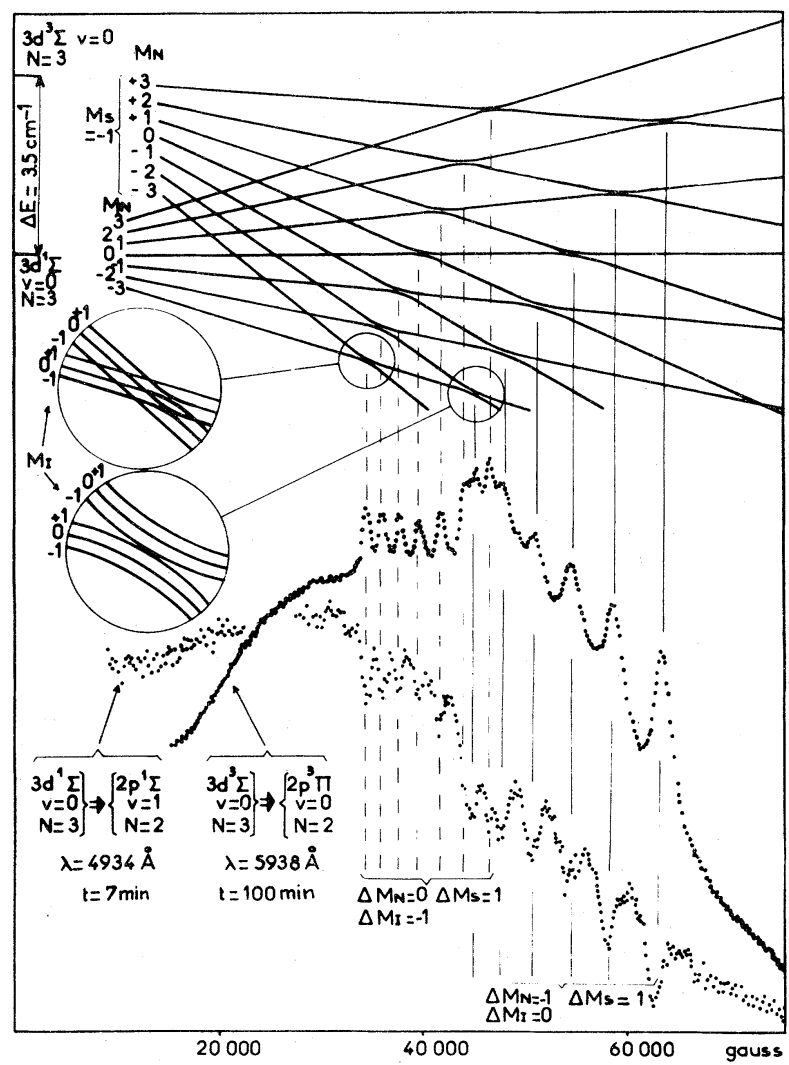

FIG. 1. Anticrossing signal both on the $4934-\AA R 2$ singlet and on the 5938- $\AA 2$ triplet ortho lines, and the corresponding Zeeman energy level diagram. The hyperfine sublevels are sketched very roughly only in the circles because fine and hyperfine structures are not known. The signal amplitude is a few percent. tion can discriminate, at least partially, between light emitted by the two levels. These levels would cross for a particular value of an external (usually magnetic) field, unless a coupling between these two levels by some repulsive interaction $V$ occurs. Roughly speaking, at the anticrossing point, the population of one level oscillates back and forth between the two levels, producing an even distribution. The resultant resonancelike signal is centered on the anticrossing point. Its amplitude is proportional both to the population difference of the two levels and to the difference of detection efficiency of the same levels. It has a width given by the field needed to separate the two levels by an amount of the order of $V$ (or the mean value of their inverse lifetime if $V$ is smaller than this quantity). In earlier experiments $V$ is due either to an electric field $7^{7-9}$ or to the hyperfine interaction within a fine or hyperfine multiplet. ${ }^{7}$ In our experiment

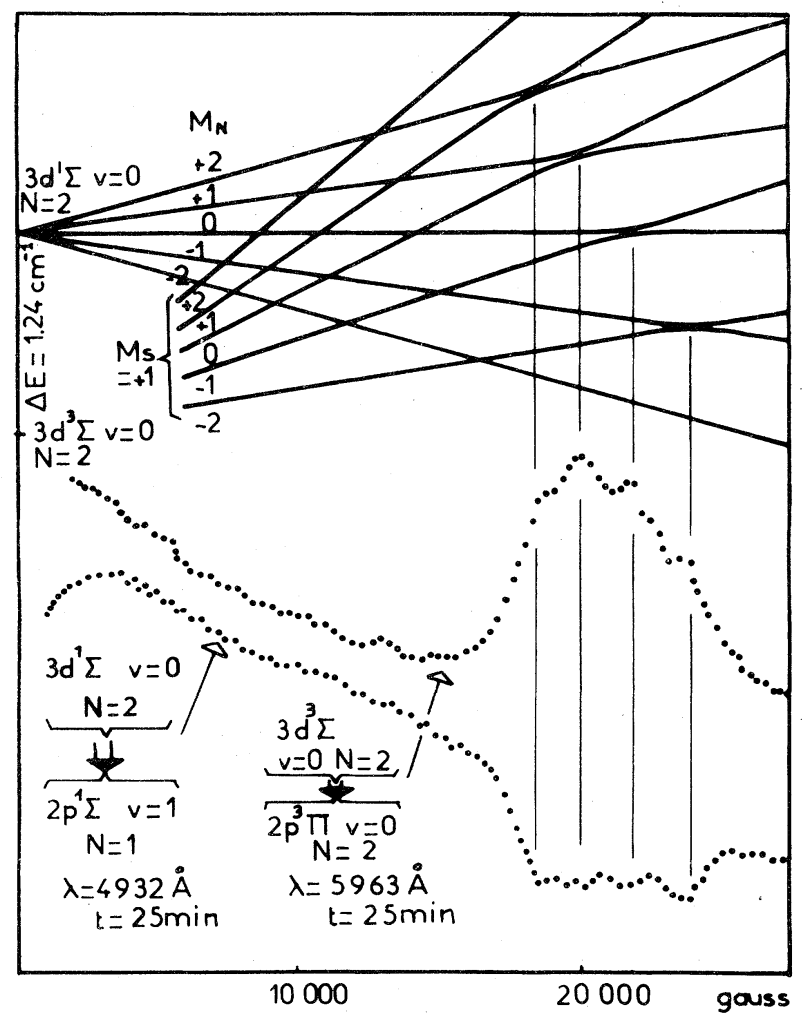

FIG. 2. Anticrossing signal both on the 5963- $\AA 2$ triplet and on the $4932-\AA R 1$ singlet para lines and the corresponding Zeeman energy level diagram. Zerofield fine structure of triplet is not known. The repartition of intensities of the various anticrossings in two lines is different because they are respectively $Q 2$ and $R 1$ lines. 
TABLE I. Zero-field separations of levels.

\begin{tabular}{ccc}
\hline \multicolumn{1}{c}{ Levels } & $\begin{array}{c}\text { Triplet minus singlet } \\
\text { separation measured } \\
\left(\mathrm{cm}^{-1}\right)\end{array}$ & $\begin{array}{c}\text { Triplet levels must } \\
\text { be lowered by }\left(\mathrm{cm}^{-1}\right)\end{array}$ \\
\hline $\begin{array}{l}3 d^{1-3} \Sigma \\
v=0, N=2 \\
3 d^{1-3} \Sigma \\
v=0 N=3\end{array}$ & $-1.24 \pm 0.1$ & 157.69 \\
$3 d^{1-3} \Delta$ & $+3.5 \pm 0.2$ & 157.75 \\
$v=1, N=3$ & $+1.16 \pm 0.1$ & 157.64 \\
\hline
\end{tabular}

the coupling is due either to spin-orbit interaction (which, as is well known, couples singlet and triplet levels, causing transition from $L S$ to $j j$ coupling) or to hyperfine Fermi contact interaction (the coupling of the analogous $3 d-4 d$ singlet and triplet levels of He by this Hamiltonian has been calculated for other purposes by Bessis, Lefebvre-Brion, and Moser ${ }^{10}$ ).

We used a $150-\mathrm{kG}$ Bitter coil. $\mathrm{H}_{2}$ was excited at a pressure of $10^{-1}$ Torr within a small Pyrex cell by a capacitive discharge (with electric field parallel to the magnetic field) induced by a $30-\mathrm{W}$, 150-MHz AMECO high-frequency generator. The intensity of the light emitted parallel to the magnetic field was detected by a EMI 9558 photomultiplier and stored on a DIDAC 800 multichannel analyzer. Between four and fifty scans of 100 sec were used. Lines were separated by a JobinYvon H.R.S. monochromator whose slit width was always great enough not to separate the Zeeman components. Figures 1 and 2 show the most characteristic results.

The position of the anticrossing gives first the zero-field separations described in Table I. These results are in good agreement and we conclude that triplet levels must by lowered by 157.7 $\pm 0.2 \mathrm{~cm}^{-1}$ as compared to Dieke's data. ${ }^{4}$ Secondly, the splitting of the various $M_{N}$ anticrossings gives the difference between orbital singlet and triplet Landé $g$ factors. Using the singlet Landé $g$ factors measured by Dieke, Cunningham, and Byrne, ${ }^{11}$ we found the following preliminary results:

$$
\begin{aligned}
& g_{N}\left(3 d^{3} \Sigma, v=0, N=2\right)=0.70 \pm 0.02, \\
& g_{N}\left(3 d^{3} \Sigma, v=0, N=3\right)=0.55 \pm 0.02 .
\end{aligned}
$$

Finally, the width of the curve can give a measure of the fine- and hyperfine-structure constants. The complete analysis of these results needs a more detailed calculation and further ex- periments are in progress which will be reported in the future. We notice that the ultimate accuracy of our method is limited by the width of the curves which is given by the fine- or hyperfinestructure constants. This is better than the Doppler-limited accuracy with which the separations within the singlet and the triplet group of levels are known. Natural-lifetime accuracy could be achieved with an existing Momrie ${ }^{9}$ apparatus, at least for the $3 d^{1-3} \Delta, v=1, N=3$ levels for which the anticrossings are at about $12 \mathrm{kG}$ : One cannot induce resonance between pure singlet and triplet levels except in the vicinity of an anticrossing point where the two levels are mixed.

We thank the Service National des Champs Intenses of the Centre National de la Recherche Scientifique at Grenoble which made available to us the 150-kG Bitter coils. We thank also the Max Planck Institute of Grenoble for the loan of optical apparatus.

\footnotetext{
*Work performed at the Service National des Champs Intenses at Grenoble (Centre National de la Recherche Scientifique).

†Laboratoire associé au Centre National de la Recherche Scientifique.

${ }^{1}$ W. Kolos and L. Wolniewicz, J. Chem. Phys. 41, 3663 (1964), and $\underline{43}, 2429$ (1965), and $\underline{45}, 509$ (1966), and $\underline{48}, 3672(1968)$.

${ }^{2} \mathrm{~S}$. Rothenberg and E. R. Davidson, J. Chem. Phys. $\underline{45}, 2560$ (1966).

${ }^{3}$ W. Kolos, Chem. Phys. Lett. 1, 19 (1967).

${ }^{4}$ G. H. Dieke, J. Mol. Spectrosc. 2, 494 (1968), and in The Hydrogen Molecule Wavelength Tables of Gerhard Heinvich Dieke, edited by H. M. Crosswhite (Wiley-Interscience, New York, 1972).

${ }^{5}$ H. Beutler and H. O. Jünger, Z. Phys. 101, 285 (1936) .

${ }^{6} \mathrm{G}$. Herzberg and Ch. Jungen, J. Mo. Spectrosc. $\underline{41}$, 425 (1972).

${ }^{7}$ H. Wieder and T. G. Eck, Phys. Rev. 153, 103 (1967);
} 
T. G. Eck, L. L. Foldy, and H. Wieder, Phys. Rev. Lett. 10, 239 (1963).

${ }^{8}$ D. H. Levy, J. Chem. Phys. 56, 5493 (1972).

${ }^{9}$ R. S. Freund and T. A. Miller, to be published.

${ }^{10}$ N. Bessis, H. Lefebvre-Brion, and C. M. Moser,
Phys. Rev. 135, A957 (1964).

${ }^{11} \mathrm{G}$. H. Dieke, S. P. Cunningham, and F. T. Byrne, Phys. Rev. 92, 81 (1953).

${ }^{12}$ M. Lombardi, J. Chem. Phys. 58, 797 (1973), and references quoted there.

\title{
Two-Center Thomas-Fermi Model for Adiabatic Ion-Atom Collisions
}

\author{
J. Eichler and U. Wille \\ Hahn-Meitner-Institut für Kernforschung Berlin GmbH, Bereich Kern- und Strahlenphysik, \\ Berlin, West Germany, and Freie Universität Berlin, Fachbereich Physik, Berlin, West Germany \\ (Received 14 May 1974)
}

\begin{abstract}
We propose a two-center model for the description of ion-atom collisions proceeding via a quasimolecular (adiabatic) mechanism. The electronic potential of the model is given by a superposition of modified atomic Thomas-Fermi potentials, each centered about one of the colliding nuclei. Molecular correlation diagrams calculated for the NeNe system show good agreement with results from molecular-orbital Hartree-Fock calculations.
\end{abstract}

In the past few years the electron-promotion model $^{1}$ has proven to be quite successful ${ }^{2}$ in describing the excitation of inner-shell electrons in adiabatic ion-atom collisions. Based on the Born-Oppenheimer approximation, the model assumes the formation of a transient quasimolecule whose electronic energy spectrum as a function of the internuclear distance ("correlation diagram") is the starting point for any dynamical treatment of the process. While for a few cases quantitative adiabatic correlation diagrams have been calculated by the Hartree-Fock method, ,34 the evaluation of most of the experimental results has been based on rather qualitative "diabatic" diagrams. They are obtained from the known electronic spectra in both the united-atom and the separated-atom limits using the correlation diagrams for unscreened Coulomb centers as a guide to connect the limiting cases. ${ }^{1,5}$

It is quite clear that for a more quantitative discussion of experiments and a detailed dynamical theory a method is needed which yields accurate adiabatic correlation diagrams like the Hartree-Fock method and yet is flexible and simple enough to be easily applicable to any given combination of collision partners.

In the present note we propose a model which we believe to meet these requirements. We start from the observation that atomic energy levels may, to a good accuracy, be derived from a statistical Thomas-Fermi (TF) model with a universal spherical single-electron potential $V^{\mathrm{TF}}(r)$ (including corrections for self-interaction and exchange effects). Latter ${ }^{6}$ has calculated energy levels for a large number of atomic states using potentials of the form (atomic units are used throughout)

$$
V^{\mathrm{TF}}(r)=\left\{\begin{array}{l}
-\frac{Z}{r}\left\{\Phi(x)+\frac{3 \sqrt{2}}{4 \pi}\left[\frac{r}{Z} \Phi(x)\right]^{1 / 2}\right\}, \text { if }\left|V^{\mathrm{TF}}(r)\right|>\frac{1}{r} \\
-r^{-1}, \text { otherwise. }
\end{array}\right.
$$

Here, $x=r / \mu$ with $\mu=0.8853 Z^{-1 / 3}$, and the screening function $\Phi(x)$ is given by the analytic expression

$$
\Phi(x)=\left(1+0.02747 x^{1 / 2}+1.243 x-0.1486 x^{3 / 2}+0.2302 x^{2}+0.007298 x^{5 / 2}+0.006944 x^{3}\right)^{-1} .
$$

The corrections for electronic self-interaction and exchange included in the potential (1) have been discussed in detail by Coulson and Sharma. ${ }^{7}$ Although the TF approximation cannot compete in accuracy with elaborate atomic Hartree-Fock calculations available today, ${ }^{8}$ it should be suf- ficiently reliable for the treatment of inner-shell electrons relevant to atomic collision processes. We here assume that the TF method not only gives a resonable description of the potential felt by a single electron in the united-atom and sep- 